\title{
Research
}

\section{The Role of Collagen Genetic Discrepancies in Development of Pelvic Organ Prolapse in Women: A Study with Negative Results}

\author{
Hirron Fernando ${ }^{2}$, Dmitry Vishnevsky ${ }^{1}$, George Kasyan ${ }^{1}$, Dmitry Pushkar $^{1}$, and Larisa Akulenko ${ }^{3}$ \\ ${ }^{1}$ Urology Department of Moscow State University of Medicine and Dentistry, Russia \\ ${ }^{2}$ James Paget University Hospital, United Kingdom \\ ${ }^{3}$ Medical Genetics Department of Moscow State University of Medicine and Dentistry, Russia
}

${ }^{\star}$ Correspondence to: Hirron Fernando; James Paget University Hospital, United Kingdom; Tel: +447818684998; Email: hirron.fernando@gmail.com

Received: Nov 1 ${ }^{\text {st }}, 2020$; Accepted: Nov 5 ${ }^{\text {th }}, 2020$; Published: Nov $7^{\text {th }}, 2020$

Citation: Fernando H, Vishnevsky D, Kasyan G, Pushkar D, and Akulenko L. The role of collagen genetic discrepancies in development of pelvic organ prolapse in women: A study with negative results. Urology Open A Open J. 2020; I(1): 12-16.

\begin{abstract}
\section{Introduction}

Pelvic organ prolapse has a mixed aetiology - hereditary and acquired. During last decade, the role of genetics in POP becomes profoundly obvious. women with a family history of prolapse are at an increased risk of prolapse refractory to treatment. Careful literature review from the past studies reveals that several genetic mutations have been shown to correlate with increased prolapse susceptibility. These mutations can result in disordered collagen metabolism, which weaken the fascial support of the pelvic organs. This prompt us to undertake the above study, look more into genetic discrepancies and pelvic organ prolapse contemporary studies relate to this topic shows that Collagen is playing a major role in pelvic floor supportive structures. However role of single nucleotide polymorphism (SNP) of the COL1A1 or COL3A1 or COL18A1 genes remain controversial relate to pelvic organ prolapse. Some studies and meta-analysis found a strict correlation between these genetic defects and POP.
\end{abstract}

\section{Methods}

Include sample size and statistical approaches.

\section{Results}

The Results must contain sufficient data for readers to evaluate the credibility of the conclusion. All of the data does not need to be presented. The conclusion should be an inference, not a summary.

\section{Conclusions}

Readers should be able to understand the question asked in the study and why and how it was done.

Keywords: Genetics; Single Nucleotide Polymorphism (SNP); Pelvic organ prolapse.

\section{INTRODUCTION}

Pelvic Organ Prolapse (POP) is the herniation of viscera into the vagina, ${ }^{1}$ generally associated with weakness of the supporting structures. Prolapse is a common condition that affect women in menopausal years. however, recent studies show that women of premenopausal years can be equally affect by this. Prolapse is likely to become more prevalent as our population ages, so a review of new evidence regarding the aetiology of this condition is timely.

Aside from the traditional risk factors such as age, parity, vaginal childbirth and increased intra-abdominal pressure (ie obesity, chronic cough 
and constipation), ${ }^{2}$ the concept of a genetic tendency towards prolapse is becoming more apparent. For example, women with a positive family history of POP are more likely to develop prolapse compared to women with no family history (OR 2.58, 95\% CI 2.12-3.15). ${ }^{3}$ Furthermore, twin studies have identified that genetic factors contribute about $43 \%$ of the variation in liability to prolapse. ${ }^{4}$

Most gynaecologists are aware of the link between connective tissue disorders and prolapse. For example, women who have collagen-associated disorders such as Ehlers-Danlos syndrome and Marfan syndrome have an increased prevalence of prolapse. Such women tend to have more severe prolapse symptoms and are more likely to get recurrence after vaginal repair. ${ }^{5-8}$ Women with a history of haemorrhoids, varicose veins, hernias and abdominal striae (conditions thought to be related to collagen deficiency) are also more likely to develop symptomatic prolapse. ${ }^{9-11}$ Conversely, women with pro-lapse are significantly more likely to develop hernias com-pared with controls ( $31.6 \%$ vs $5 \%$, $\mathrm{n}=20, P=0.002) .{ }^{12}$ There is strong evidence that collagen imbalance contributes to the pathogenesis of hernias. ${ }^{13}$

Changes in collagen metabolism may lead to weakened fascia, which plays an essential role in the support of the pelvic viscera. ${ }^{14}$ Over the past five years, studies have identified several genetic variants (or polymorphisms) implicated in prolapse. ${ }^{15}$ These genetic variations affect collagen synthesis and remodelling of the extracellular matrix (ECM)

.${ }^{16}$ This review aims to summarise recent findings regarding the role of collagen dysfunction and its relation to genetic aberrations in the pathogenesis of POP. ${ }^{17}$

\section{MATERIALS AND METHODS}

This case-control trial investigated the prevalence of COL1A1, COL3A1 and COL18A1 genes SNP in female patients suffering with POP in comparison with women without these symptoms. The number of the patients in the study and control groups were in 1,5:1 ratio. Inclusion criteria a study and control groups were limited to having at least one or more POP risk factors, such as: two or more births through natural paths, traumatic births, births of more than 4,000 grams, excessive physical activity, diseases accompanied by increased intraabdominal pressure (bronchial asthma, chronic bronchitis, chronic constipation), history of pelvic surgery. Exclusion criteria for both groups were hereditary diseases with a known increased risk of POP, such as Marfan or EhlersDanlos syndrome as well as previous surgeries for POP/SUI for control group. All women included for both treatment and control groups were Caucasian of Russian descent.

The trial was approved by Local Ethics Committee and registered in International Clinical Trials Registry Platform (N 34678).

\section{Laboratory Tests}

Participants gave a saliva sample in order to perform a genetic test. The medical history was collected and filed; pelvic floor physical exam will be done. The saliva samples will be analyzed for SNP in COL3A1 (rs1800255, rs1801184, rs111929073); in COL1A1 (rs1800012); in COL18A1 (rs2236479). Genotyping was performed by Sanger sequencing method, [8] followed by estimation of sensitivity and specificity for POP and SUI.

Primers specificity was confirmed by polymerase chain reaction (PCR) with subsequent electrophoresis in agarose gel. Sanger se- quence of PCR product was performed on ABI 3730XL (Life Technologies) from one end, one reaction per template on ABI.

To determine the correlation of pelvic floor dysfunction with a combination of polymorphisms rs1800012, rs1800255, rs2236479, rs1801184 the method of logistic regression with the inclusion of all independent factors was used.

\section{RESULTS}

The study group included 150 Caucasian women with POP (mean age 64.4years, from 40 to 70 years old). One hundred women matched with age and risk factors but without POP were included as a control group.

The COL1A1 gene encodes the alpha-1 protein of the type I collagen chain. A section of DNA in the regulatory region of the COL1A1 gene at position 1245 , in which guanine $(G)$ is replaced by thymine (T), was investigated. As a result of substitution, the nature of gene transcription changes and the normal ratio of collagen chains is violated, which leads to a violation of the fiber structure.

The gene COL1A1 at the locus $+1245 \mathrm{G}>\mathrm{T}$ (rs1800012) has 2 alleles: G-major allele (main) and T-minor allele (replacement, mutation). The $\mathrm{T}$ allele is associated with increased transcription of the type 1 collagen gene and is associated with many connective tissue disorders. The incidence of the $\mathrm{T}$ allele in the European population is $20 \%$. Possible marker genotypes: G/G; G/T; T / T. The results of the comparative analysis of the frequency distribution of alleles and genotypes of the polymorphic variant $+1245 \mathrm{G}>\mathrm{T}$ ( $\mathrm{rs} 1800012)$ of the COL1A1 gene in the study and control groups showed that carriers of the minor allele" $\mathrm{T}$ " polymorphism rs 1800012 gene COL1A1 were $28 \%$ of patients with POP (26\% heterozygous and $2 \%$ homozygous genotypes) and $33 \%$ of patients in the control group (32\% heterozygous and $1 \%$ homozygous genotypes).

To determine the significance of the polymorphic variant 2209G >A (R. Ala698Thr, rs1800255) in the a-chain gene COL3A1 in the development of DTE, we conducted a comparative analysis of allele frequencies and genotypes in the study and control groups. Gene COL3A1 locus 2209G >A (R. Ala698Thr, rs1800255) has 2 alleles: major (main) allele $\mathrm{G}$ and minor (replacement) allele A. Possible marker genotypes: G/G; G/A; A / A. Results of comparative analysis of frequency distribution and genotypes of polymorphic variant $2209 \mathrm{G}>\mathrm{A}$ (R. Ala698Thr, rs1800255) of gene COL3A1 revealed that carriers of the minor allele A polymorphism rs 1800255 gene COL3A1 were $36 \%$ of patients with POP $(29.3 \%$ heterozygous and $6.7 \%$ homozygous genotypes) and $36 \%$ of patients in the control group ( $28 \%$ heterozygous and $8 \%$ homozygous genotypes).

With the same manner, we found no difference in the polymorphic variant rs1801184 and rs111929073 in the type 3 collagen a-chain gene (COL3A1) in patients with POP and control group.

A polymorphic variant of $\mathrm{rs} 2236479(\mathrm{G}>\mathrm{A})$ of the COL18A1 gene was also considered. The COL18A1 gene at the rs2236479 locus has 2 alleles-major allele-G and minor allele-A. Possible genotypes of this marker are G\G; G\A; GA. There was no statistically significant difference between groups in it as well.

A logistic regression with forced inclusion of all independent factors was conducted. As a dependent variable, the patient's belonging to the study or control group was used. The binary variables of genetic polymorphisms presence were used as independent variables: 
rs800012, rs1800255, rs2236479, rs1801184. There were no association of the combination of the studied polymorphisms and POP

\section{STATISTICAL METHODS}

Statistical data processing was performed using the PASW Statistics 22 program.

Descriptive statistics were presented in the form of mean and standard deviation, median and 25th and 75th percentiles, minimum and maximum values in the sample for quantitative variables, and frequency of occurrence and proportion in the sample for qualitative variables.

Prior to the construction of the predictive model, independent parameters were compared in two studied Groups in order to determine the set of parameters that are most suitable for inclusion in the multi-factor model. The Mann-Whitney U-test was used to compare quantitative UES In two unrelated samples. The exact Fischer test was used to compare nominal variables in two unrelated populations. The significance level (p) was assumed to be 0.05 in all the above comparisons.

After conducting a preliminary analysis, variables whose occurrence in the total sample was less than $10 \%$ were excluded from the list of independent factors participating in the model, taking into account the absence of statistically significant differences in the Overall indicators in the two studied populations.

Taking into account the presence of significant differences between the two studied Groups in the quantitative variable "Age", the ESN variable was reduced to a categorical form for its inclusion in the multi-factor predictive model. In order to find the condition for dividing the variable "Age" into 2 categories, we used the method of constructing classification trees using the CART algorithm.

Taking into account the available literature, as well as the above conditions for the frequency of occurrence of variables in the General sample, 8 categorical variables were included in the initial model.

To build a multi-factor predictive model, we used the method of logistic regression with step-by-step elimination of variables using the Values algorithm. The step selection criteria were assumed to be between 0.01 and 0.45 . Model compliance statistics were evaluated using the Omnibus test for model coefficients, as well as the Hosmer-Lemeshev test at each step of the model. The subject's membership in the Study or control group was used as a dependent variable. Categorical variables that meet the selection conditions described above were used as independent variables. The regression equation has the form: $y=\exp \left(b 0+b 1^{*} x 1+\ldots\right.$ $\left.+b n^{\star} x n\right) /\left[1+\exp \left(b 0+b 1^{\star} x 1+\ldots+b n^{*} x n\right)\right]$,

$$
\begin{aligned}
& \mathrm{xi} \text { - independent variable, } \\
& \mathrm{y} \text { - dependent binary variable } \\
& \text { bi - regression coefficient }
\end{aligned}
$$

A classification table was constructed for initial (zero) and at the last step of the regression algorithm with a fixed threshold classification $=0.50$. To find the optimal threshold classification sensitivity and specificity was found to be of prognostic models were constructed ROC curve, where the check variable was used, the probability of the predicted values as a state variable - Study group. Descriptive statistics. [Table 1,2].

\begin{tabular}{|c|c|c|c|c|}
\hline & & & \multicolumn{2}{|c|}{ Group } \\
\hline & & & Controls & Study \\
\hline \multirow{8}{*}{ Age } & \multicolumn{2}{|c|}{ Valid N } & 100 & 150 \\
\hline & \multicolumn{2}{|c|}{ Mean } & 54.7 & 57.3 \\
\hline & \multicolumn{2}{|c|}{ Standard Deviation } & 9.2 & 8.6 \\
\hline & \multicolumn{2}{|c|}{ Maximum } & 70 & 70 \\
\hline & \multicolumn{2}{|c|}{ Minimum } & 40 & 40 \\
\hline & \multicolumn{2}{|c|}{ Median } & 55 & 58.5 \\
\hline & \multicolumn{2}{|c|}{ Percentile 25} & 46 & 51 \\
\hline & \multicolumn{2}{|c|}{ Percentile 75} & 62 & 64 \\
\hline \multirow{4}{*}{$\begin{array}{l}\text { Pelvic organ prolapse } \\
\text { (POP) }\end{array}$} & \multirow{2}{*}{ No } & Count & 100 & 58 \\
\hline & & Column N \% & $100.00 \%$ & $38.70 \%$ \\
\hline & \multirow{2}{*}{ Yes } & Count & 0 & 92 \\
\hline & & Column N \% & $0.00 \%$ & $61.30 \%$ \\
\hline \multirow{4}{*}{$\mathrm{POP}+\mathrm{OAB}$} & \multirow{2}{*}{ No } & Count & 100 & 36 \\
\hline & & Column N \% & $100.00 \%$ & $24.00 \%$ \\
\hline & \multirow{2}{*}{ Yes } & Count & 0 & 114 \\
\hline & & Column N \% & $0.00 \%$ & $76.00 \%$ \\
\hline \multirow{4}{*}{$\mathrm{POP}+\mathrm{SUI}$} & \multirow{2}{*}{ No } & Count & 100 & 93 \\
\hline & & Column N \% & $100.00 \%$ & $62.00 \%$ \\
\hline & \multirow{2}{*}{ Yes } & Count & 0 & 57 \\
\hline & & Column N \% & $0.00 \%$ & $38.00 \%$ \\
\hline \multirow{4}{*}{ Obesity } & No & Count & 86 & 116 \\
\hline & 100 & Column N \% & $86.00 \%$ & $77.30 \%$ \\
\hline & & Count & 14 & 34 \\
\hline & Yes & Column N \% & $14.00 \%$ & $22.70 \%$ \\
\hline & & Count & 74 & 110 \\
\hline Pelvic floer curgery & No & Column N \% & $74.00 \%$ & $73.30 \%$ \\
\hline Pelvic lioor surgery & & Count & 26 & 40 \\
\hline & Yes & Column N \% & $26.00 \%$ & $26.70 \%$ \\
\hline & & Count & 44 & 82 \\
\hline & No & Column N \% & $44.00 \%$ & $54.70 \%$ \\
\hline Constipation & Yes & Count & 56 & 68 \\
\hline & res & Column N \% & $56.00 \%$ & $45.30 \%$ \\
\hline & $\mathrm{No}$ & Count & 29 & 35 \\
\hline Menonause & NO & Column N \% & $29.00 \%$ & $23.30 \%$ \\
\hline Menopause & & Count & 71 & 115 \\
\hline & Yes & Column N \% & $71.00 \%$ & $76.70 \%$ \\
\hline & $\mathrm{No}$ & Count & 58 & 94 \\
\hline Chronic pulmonary & No & Column N \% & $58.00 \%$ & $62.70 \%$ \\
\hline disorders & Yes & Count & 42 & 56 \\
\hline & Yes & Column N \% & $42.00 \%$ & $37.30 \%$ \\
\hline & $\mathrm{No}$ & Count & 36 & 56 \\
\hline More than 2 deliveries & NO & Column N \% & $36.00 \%$ & $37.30 \%$ \\
\hline More than $\angle$ deliverles & Yes & Count & 64 & 94 \\
\hline & Yes & Column N \% & $64.00 \%$ & $62.70 \%$ \\
\hline & & Count & 59 & 72 \\
\hline Traumatic deliyery & No & Column N \% & $59.00 \%$ & $48.00 \%$ \\
\hline Iraumatic denvery & Yoct & Count & 41 & 78 \\
\hline & Yes & Column N \% & $41.00 \%$ & $52.00 \%$ \\
\hline & $\mathrm{No}$ & Count & 68 & 111 \\
\hline 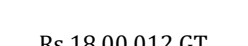 & No & Column N \% & $68.00 \%$ & $74.00 \%$ \\
\hline RS $18,00,012$ G1 & & Count & 32 & 39 \\
\hline & Yes & Column N \% & $32.00 \%$ & $26.00 \%$ \\
\hline
\end{tabular}

Table 1. A classification table was constructed for initial (zero) and at the last step of the regression algorithm with a fixed threshold classification $=0.50$ 


\begin{tabular}{|c|c|c|c|c|}
\hline \multirow{4}{*}{ Rs $18,00,255$ AG } & \multirow{2}{*}{ No } & Count & 72 & 106 \\
\hline & & Column N \% & $72.00 \%$ & $70.70 \%$ \\
\hline & \multirow{2}{*}{ Yes } & Count & 28 & 44 \\
\hline & & Column N \% & $28.00 \%$ & $29.30 \%$ \\
\hline \multirow{4}{*}{ Rs 2,23,6479 AG } & \multirow{2}{*}{ No } & Count & 76 & 127 \\
\hline & & Column N \% & $76.00 \%$ & $84.70 \%$ \\
\hline & \multirow{2}{*}{ Yes } & Count & 24 & 23 \\
\hline & & Column N \% & $24.00 \%$ & $15.30 \%$ \\
\hline \multirow{4}{*}{ Rs $18,01,184 \mathrm{TC}$} & \multirow{2}{*}{ No } & Count & 61 & 92 \\
\hline & & Column N \% & $61.00 \%$ & $61.30 \%$ \\
\hline & \multirow{2}{*}{ Yes } & Count & 39 & 58 \\
\hline & & Column N \% & $39.00 \%$ & $38.70 \%$ \\
\hline \multirow{4}{*}{ Rs $18,00,012 \mathrm{TT}$} & \multirow{2}{*}{ No } & Count & 99 & 145 \\
\hline & & Column N \% & $99.00 \%$ & $96.70 \%$ \\
\hline & \multirow{2}{*}{ Yes } & Count & 1 & 5 \\
\hline & & Column N \% & $1.00 \%$ & $3.30 \%$ \\
\hline \multirow{4}{*}{ Rs $18,00,255 \mathrm{AA}$} & \multirow{2}{*}{ No } & Count & 92 & 140 \\
\hline & & Column N \% & $92.00 \%$ & $93.30 \%$ \\
\hline & \multirow{2}{*}{ Yes } & Count & 8 & 10 \\
\hline & & Column N \% & $8.00 \%$ & $6.70 \%$ \\
\hline \multirow{4}{*}{ Rs $22,36,479$ AA } & \multirow{2}{*}{ No } & Count & 94 & 139 \\
\hline & & Column N \% & $94.00 \%$ & $92.70 \%$ \\
\hline & \multirow{2}{*}{ Yes } & Count & 6 & 11 \\
\hline & & Column N \% & $6.00 \%$ & $7.30 \%$ \\
\hline \multirow{4}{*}{ Rs $18,01,184$ CC } & \multirow{2}{*}{ No } & Count & 96 & 141 \\
\hline & & Column N \% & $96.00 \%$ & $94.00 \%$ \\
\hline & \multirow{2}{*}{ Yes } & Count & 4 & 9 \\
\hline & & Column N \% & $4.00 \%$ & $6.00 \%$ \\
\hline \multirow{4}{*}{ Rs $11,19,29,073 \mathrm{TT}$} & \multirow{2}{*}{ No } & Count & 100 & 150 \\
\hline & & Column N \% & $100.00 \%$ & $100.00 \%$ \\
\hline & \multirow{2}{*}{ Yes } & Count & 0 & 0 \\
\hline & & Column N \% & $0.00 \%$ & $0.00 \%$ \\
\hline \multirow{4}{*}{ Rs $11,19,29,073 \mathrm{TG}$} & \multirow{2}{*}{ No } & Count & 100 & 150 \\
\hline & & Column N \% & $100.00 \%$ & $100.00 \%$ \\
\hline & \multirow{2}{*}{ Yes } & Count & 0 & 0 \\
\hline & & Column N \% & $0.00 \%$ & $0.00 \%$ \\
\hline
\end{tabular}

* Variables marked with a yellow marker are excluded from further analysis due to the low frequency of occurrence in the General sample.

Table 2. Comparison of parameters in two independent sets Quality parameters. The exact Fisher criterion

\begin{tabular}{|c|c|c|c|c|c|}
\hline \multicolumn{6}{|c|}{ Crosstab } \\
\hline & & & \multicolumn{2}{|c|}{ Group } & \multirow{2}{*}{ Total } \\
\hline & & & Control & Study & \\
\hline \multirow{4}{*}{ POP } & \multirow{2}{*}{ No } & Count & 100 & 58 & 158 \\
\hline & & $\%$ within Group & $100.00 \%$ & $38.70 \%$ & $63.20 \%$ \\
\hline & \multirow{2}{*}{ Yes } & Count & 0 & 92 & 92 \\
\hline & & $\%$ within Group & $0.00 \%$ & $61.30 \%$ & $36.80 \%$ \\
\hline \multirow{2}{*}{\multicolumn{2}{|c|}{ Total }} & Count & 100 & 150 & 250 \\
\hline & & $\%$ within Group & $100.00 \%$ & $100.00 \%$ & $100.00 \%$ \\
\hline
\end{tabular}

\begin{tabular}{|c|c|c|c|c|c|}
\hline \multicolumn{7}{|c|}{ Chi-Square Tests } \\
\hline & Value & df & $\begin{array}{c}\text { Asymp. Sig. } \\
\text { (2-sided) }\end{array}$ & $\begin{array}{c}\text { Exact Sig. } \\
\text { (2-sided) }\end{array}$ & $\begin{array}{c}\text { Exact Sig. } \\
\text { (1-sided) }\end{array}$ \\
\hline Pearson Chi-Square & 97.046 & 1 & 0 & & \\
\hline Continuity Correction & 94.427 & 1 & 0 & & \\
\hline Likelihood Ratio & 128.771 & 1 & 0 & & 0 \\
\hline Fisher's Exact Test & & & & 0 & \\
\hline $\begin{array}{c}\text { Linear-by-Linear As- } \\
\text { sociation }\end{array}$ & 96.658 & 1 & 0 & & \\
\hline
\end{tabular}

\section{CONCLUSION}

Our study shows that single nucleotide polymorphisms polymorphisms in COL3A1 (rs1800255, rs1801184, rs111929073), in COL1A1 (rs1800012), and in COL18A1 (rs2236479) as well as their combinations were not associated with female POP. We draw some conclusions from our study, that further research into POP is deem necessary to ascertain if there is link between genetics and environmental factors POP.

\section{CONFLICTS OF INTEREST}

None.

\section{REFERENCES}

1. Smith FJ, Holman CD, Moorin RE, Tsokos N. Lifetime Risk of Undergoing Surgery for Pelvic Organ Prolapse. Obstet Gynecol. 2010; 116: 1096-1100. doi: 10.1097/AOG.0b013e3181f73729

2. Dietz H. The aetiology of prolapse. Int Urogynecol J Pelvic Floor Dysfunct. 2008; 19: 1323-1329. doi: 10.1007/s00192-008-0695-7

3. Lince SL, van Kempen LC, Vierhout M, Kluivers KB. A Systematic Review of Clinical Studies on Hereditary Factors in Pelvic Organ Prolapse. Int Urogynecol J. 2012; 23: 1327-1336.

4. Altman D, Forsman M, Falconer C, Lichtenstein P. Genetic Influence on Stress Urinary Incontinence and Pelvic Organ Prolapse. Eur Urol. 2008; 54: 918-22. doi: 10.1016/j.eururo.2007.12.004

5. Norton PA, Baker JE, Sharp HC, Warenski JC. Genitourinary Prolapse and Joint Hypermobility in Women. Obstet Gynecol. 1995; 85: 225-228. doi: 10.1016/0029-7844(94)00386-R

6. Carley ME, Schaffer J. Urinary Incontinence and Pelvic Organ Prolapse in Women with Marfan or Ehlers-Danlos Syndrome. Am J Obstet Gynecol. 2000; 182: 1021-1023. doi: 10.1067/mob.2000.105410

7. Aydeniz A, Dikensoy E, Cebesoy B. The Relation Between Genitourinary Prolapse and Joint Hypermobility In Turkish Women. Arch Gynecol Obstet. 2010; 281: 301-304. doi: 10.1007/s00404-009-1103-3

8. Knuuti E, Kauppila S, Kotila V. Genitourinary Prolapse and Joint Hypermobility are Associated with Altered Type I And Iii Collagen Metabolism. Arch Gynecol Obstet. 2011; 283: 1081-1085. doi: 10.1007/ s00404-010-1518-x

9. Miedel A, Tegerstedt G, Maehle-Schmidt M. Nonobstetric Risk Factors for Symptomatic Pelvic Organ Prolapse. Obstet Gynecol. 2009; 113: 1089-1097. doi: 10.1097/AOG.0b013e3181a11a85

10. Salter SA, Batra RS, Rohrer TE. Striae and Pelvic Relaxation: Two 
Disorders of Connective Tissue With A Strong Association. J Invest Dermatol. 2006; 126: 1745-1748. doi: 10.1038/sj.jid.5700258

11. Segev Y, Auslender R, Feiner B, Feiner, Arie Lissak, Ofer Lavie, et al. Are Women with Pelvic Organ Prolapse At A Higher Risk Of Developing Hernias? Int Urogynecol J Pelvic Floor Dysfunct. 2009; 20: 1451-1453. doi: 10.1007/s00192-009-0968-9

12. Henriksen NA, Yadete DH, Sorensen LT. Connective Tissue Alteration in Abdominal Wall Hernia. Br J Surg. 2011; 98: 210-219. doi: 10.1002/bjs.7339

13. Twiss C, Triaca V, Rodriguez LV. Familial Transmission of Urogenital Prolapse and Incontinence. Curr Opin Obstet Gynecol. 2007; 19: 464-468. doi: 10.1097/GCO.0b013e3282efdc21

14. Rajeev Ramanah, Bernard Parratte, Francine Arbez-Gindre, Robert Maillet, Didier Riethmuller. The Uterosacral Complex: Ligament or
Neurovascular Pathway? Anatomical and Histological Study of Fetuses and Adults. Int Urogynecol J Pelvic Floor Dysfunct. 2008; 19: 1565-1570. doi: 10.1007/s00192-008-0692-x

15. Emily E Cole, Patrick B Leu, Alex Gomelsky, Patricia Revelo, Heidi Shappell, et al. Histopathological Evaluation of The Uterosacral Ligament: Is This A Dependable Structure for Pelvic Reconstruction. BJU Int. 2006; 97: 345-348. doi: 10.1111/j.1464-410X.2005.05903.x

16. S A Butler-Manuel, L D Buttery, R P A'Hern, J M Polak, D P Barton. Pelvic nerve Plexus Trauma at Radical Hysterectomy and Simple Hysterectomy - The Nerve Content of The Uterine Supporting Ligaments. Cancer. 2000; 89: 834-841. doi: 10.1002/1097-0142(20000815)89:4<834::aidcncr 16>3.0.co;2-7

17. DeLancey JO. The anatomy of the pelvic floor. Curr Opin Obstet Gynecol. 1994; 6: 313-316. 